\title{
FACTORES PSICOLÓGICOS EN LA ADAPTACIÓN DE PACIENTES SOMETIDOS AL PROCESO DE CONSEJO GENÉTICO PARA CÁNCER DE MAMA Y OVARIO HEREDITARIO
}

\author{
Autora: Melinda González Concepción \\ Tipo de trabajo: Tesis Doctoral. \\ Directores: Tomás Blasco e Ignacio Blanco. \\ Centro: Universidad Autónoma de Barcelona. Facultad de Psicología. Departa- \\ mento de Psicología Básica, Evolutiva y de la Educación.
}

Fechas de lectura: 21 de Julio de 2016.

E- mail: gcmelinda@gmail.com

Introducción. Entre el $5-10 \%$ de todos los cánceres se produce por mutaciones heredadas que se trasmiten de padres a hijos y de generación en generación. Estas mutaciones determinan que el individuo tenga una probabilidad mucho mayor que la población general de desarrollar cáncer. Además, este cáncer puede aparecer a edades tempranas. En estos casos hablamos de cáncer hereditario, para cuya detección y manejo se han desarrollado las Unidades de Consejo Genético (UCG). En los pacientes con cáncer que son portadores de las mutaciones, la UCG plantea llevar a cabo un estudio para identificar a los familiares sanos que puedan poseer también dichas mutaciones y proponerles estrategias personalizadas que les permitan prevenir, detectar y tratar de manera precoz la posible enfermedad oncológica para la cual su riesgo es superior al del resto de la población. Así pues, las personas acuden a la UCG en busca de asesoramiento para su situación. Sin embargo, la incertidumbre y el riesgo del proceso de consejo genético puede dar lugar a un malestar emocional que es necesario conocer a fondo para poderlo manejar de la forma más eficaz posible
Objetivo: Analizar si en el proceso de consejo genético para pacientes portadoras del gen BRCA1/2 responsable del síndrome de cáncer de mama y ovario hereditario $(\mathrm{CMOH})$, la Percepción de Control (evaluada a partir de una adaptación de la escala Perceived Personal Control) puede determinar, y en qué grado: a) El Bienestar Psicológico (evaluado con la escala de Ryff); b) La existencia de Cambios Positivos (Benefit Finding); y, c) La realización correcta de las conductas de prevención y vigilancia establecidas por la UCG.

Método: Se solicitó a las pacientes atendidas en el Programa de Cáncer Hereditario del Institut Catala d'Oncologia y que recibieron visita de seguimiento entre los meses de Julio 2012 y Mayo 2014 en las diferentes unidades del programa, su colaboración para el estudio. Para ello debían cumplimentar, en su domicilio, una batería de cuestionarios que recogía los aspectos antes citados, junto con otras informaciones sobre su vivencia de la enfermedad (Preocupación por el Cáncer, evaluada mediante la Cancer Worry Scale) y las características de la misma. La muestra final estuvo for- 
mada por 176 pacientes (con edades comprendidas entre 24 y 81 años), de las cuales 98 eran portadoras afectas de $\mathrm{CMOH}$ y 78 eran portadoras sanas.

Resultados: El primer resultado de este trabajo ha sido la elaboración de la versión española de la escala Perceived Personal Control. Dicha adaptación mantiene 6 de los 9 ítems de la escala original y tiene una estructura unidimensional con una fiabilidad satisfactoria (Alfa de Cronbach $=0,84$ ). A partir de dicha escala, los datos del estudio revelan que la Percepción de Control guarda una relación directa con cuatro de las seis dimensiones que componen el Bienestar Psicológico. Respecto a la existencia de Cambios Positivos, dicha existencia tenía una relación inversa con el nivel de Preocupación por el Cáncer, pero no mostró relaciones con la Percepción de Control. Sin embargo, las pacientes sí indicaban que el acudir a la UCG era una experiencia positiva pues permitía saber que existía la posibilidad de detectar y tratar precozmente la enfermedad oncológica. Finalmente, se observó que el grado de seguimiento correcto de las conductas de prevención y vigilancia de las pacientes era muy escaso, y tampoco se relacionaba con la Percepción de Control.

Conclusiones: La adaptación española de la escala de Percepción de
Control permite valorar el grado de control percibido por las pacientes en el Proceso de Consejo Genético. Los resultados indican que las pacientes consideran como una consecuencia positiva el asesoramiento recibido en la UCG pues les permite tener sensación de control sobre la detección y el tratamiento precoz de la enfermedad oncológica. El malestar emocional está determinado en parte por la Percepción de Control, pero también por otras variables como el riesgo percibido o el nivel de preocupación por el cáncer. Sin embargo, la adhesión a conductas preventivas no parece depender de la percepción de control de las pacientes. Las implicaciones clínicas de estos resultados apuntan a que las UCG ayudan a manejar el malestar emocional a través de un incremento de la Percepción de Control en las pacientes, por lo que puede considerarse que los protocolos de actuación son adecuados. Sin embargo, debe incidirse en las conductas de prevención, ya que el seguimiento de las mismas por parte de las pacientes es todavía insatisfactorio. Nuestros resultados no permiten detectar a qué puede deberse este escaso seguimiento, por lo que deben hacerse investigaciones que identifiquen las variables que regulan estos comportamientos. 